\title{
Reference Point Group Mobility and Random Waypoint Models in Performance Evaluation of MANET Routing Protocols
}

\author{
Geetha Jayakumar and Gopinath Ganapathi \\ Department of Computer Sciences, Bharathidasan University, Tiruchirappalli 620 023, Tamil Nadu, India
}

Correspondence should be addressed to Geetha Jayakumar, j.geetha@gmail.com

Received 7 August 2008; Revised 29 September 2008; Accepted 5 December 2008

Recommended by Walaa Hamouda

\begin{abstract}
Ad hoc networks are characterized by multihop wireless connectivity, frequently changing network topology and the need for efficient dynamic routing protocols plays an important role. We compare the performance of two prominent on-demand routing protocols for mobile ad hoc networks: dynamic source routing (DSR), ad hoc on-demand distance vector routing (AODV). A detailed simulation model with medium access control (MAC) and physical layer models is used to study the interlayer interactions and their performance implications. We demonstrate that even though DSR and AODV share similar on-demand behavior, the differences in the protocol mechanisms can lead to significant performance differentials. In this paper, we examine both ondemand routing protocols AODV and DSR based on packet delivery ratio, normalized routing load, normalized MAC load, average end-to-end delay by varying the node density, network loading, and mobility variations for reference point group mobility and random waypoint models. This framework aims to evaluate the effect of mobility models on the performance of mobile ad hoc networks (MANETs) routing protocols. Our results show that the protocol performance may vary drastically across mobility models and performance rankings of protocols may vary with the mobility models used. This effect can be explained by the interaction of the mobility characteristics with the connectivity graph properties.
\end{abstract}

Copyright (C) 2008 G. Jayakumar and G. Ganapathi. This is an open access article distributed under the Creative Commons Attribution License, which permits unrestricted use, distribution, and reproduction in any medium, provided the original work is properly cited.

\section{INTRODUCTION}

Wireless technologies are without any doubt shaping the beginning of the new millennium. Cellular networks $(2 / 2.5 / 3$ Generations) have reached global coverage and wireless LANs are providing high speed internet access to city center and hot spot areas. The principle of computing anytime anywhere is becoming reality. Consequently, there has been tremendous interest recently in the convergence of wired (fixed) networks and mobile wireless and local wireless access systems and networks. Beyond 3G (B3G) and 4G concepts have been the very hot subject of much research and many standardization activities throughout the world. Many B3G/4G-related technical committees are taking the initiative to produce enhanced proposals of radio transmission technologies and wireless applications protocols [1].

Computer network, traditionally viewed as infrastructure of a fixed form, has evolved into combinations of wired and wireless networks to suit today's need of mobile communication. As the mobility of users continues to increase, a special type of network will be gaining more and more attention which is mobile ad hoc network. Such research on the capacity of wireless ad hoc networks and the popular IEEE 802.11 protocol typically assume the use of omnidirectional antennas at all nodes [2]. Over the past few years, considerable amount of research addressed the issues, such as, (a) access, (b) routing, (c) transport layer protocols, (d) quality of service provisioning, (e) selforganization, and (f) energy management. Recent research includes understanding the cross-layer protocol interactions and new designs of such protocols $[3,4]$.

In a mobile ad hoc network, nodes do not rely on any existing infrastructure. Instead, the nodes themselves form the network and communicate through means of wireless communications. Mobility causes frequent topology changes and may break existing paths. A routing protocol should quickly adapt to the topology changes and efficiently 
search for new paths. On the other hand, the limited power and bandwidth resources in mobile ad hoc networks make quick adaptation very challenging [5]. Nodes will have to forward the network traffic on behalf of other nodes to allow communication to take place between nodes that are out of each other immediate radio range. Hence, routing of network traffic becomes a central issue in these networks. The mobile ad hoc networks are envisioned to support dynamic and rapidly changing multihop topologies which are likely to be composed of relatively bandwidth constrained wireless links [6]. A generic framework to systematically analyze the impact of mobility on the performance of routing protocols for MANET has become important. It is necessary to find out what degree of mobility affects routing protocol performance. Many previous studies have used random waypoint (RWP) as reference model [7]. Random waypoint is a simple model that is easy to analyze and implement. This has probably been the main reason for the widespread use of this model for simulations. Further, the RWP model is not sufficient to capture some realistic scenarios. In order to model the movements of nodes in a realistic terrain, such as a battlefield or rescue operation, some sophisticated mobility models such as reference point group mobility (RPGM) models find valuable application.

In the RWP model, the nodes, that is, mobile users, move along a zigzag path consisting of straight legs from one waypoint to the next [8]. Mobility model such as RWP model is described on graphs based on road maps [9].

For this purpose, numerous ad hoc routing protocols have been proposed by the Internet Engineering Task Force (IETF) working group MANET. These protocols have been evaluated through simulations in network simulators like NS-2.

\section{BACKGROUND}

The routing protocols for MANET can be broadly classified as on-demand/reactive and periodic/proactive protocols. Reactive routing protocols propagate route updates only when a route to a destination is required. There are several reactive routing protocols available for ad hoc networks, including dynamic source routing (DSR) [10], ad hoc ondemand distance vector (AODV) [11], and so on. Reactive routing protocols have been demonstrated to perform better with significantly lower overheads than proactive routing protocols in many scenarios [12] since they are able to react quickly to topology changes, yet being able to reduce routing overhead in periods or areas of the network in which changes are less frequent. In this section, we briefly discuss the working of both on-demand routing protocols AODV and DSR. Their respective performances are compared in Section 1. It has also been observed that under a given mobility pattern, routing protocols like DSR and AODV perform differently.

This is possibly because each protocol differs in the basic mechanisms or "building blocks" it uses. For example, DSR uses route discovery. To find out how, we need to investigate the effect of mobility on some of these "building blocks" and how they impact the protocol performance as a whole.
It is necessary to systematically propose a framework to analyze the impact of mobility on the performance of routing protocols in ad hoc networks. Through this framework, we illustrate how modeling mobility is important in affecting routing performance and understanding the mechanism of ad hoc routing protocols. Our framework mainly focuses on the following aspects: mobility models, the metrics for mobility and connectivity graph characteristics, the potential relationship between mobility and routing performance, and the analysis of impact of mobility on building blocks of ad hoc routing protocols.

\subsection{Reactive protocols}

In contrast to table-driven routing protocols, all up-todate routes are not maintained at every node; instead the routes are created as and when required. When a node requires a route to the destination, it invokes a route discovery mechanism to find the path to the destination. The process is completed once a route is found or all possible route permutations have been examined. Once a route has been established, it is maintained by a route maintenance procedure until either the destination becomes inaccessible along every path from the source or until the route is no longer desired. This section discusses two on-demand routing protocols.

\section{2. $A O D V$}

AODV (see [11]) is an improvement on the destinationsequenced distance vector (DSDV). AODV uses an ondemand approach for finding routes. Since it is an ondemand algorithm, a route is established only when it is required by a source node for transmitting data packets and it maintains these routes as long as they are needed by the sources. AODV uses a destination sequence number, created by the destination, to determine an up-to-date path to the destination. A node updates its route information only if the destination sequence number of the current received packet is greater than the destination sequence number stored at the node. It indicates the freshness of the route accepted by the source. To prevent multiple broadcast of the same packet, AODV uses broadcast identifier number that ensures loop freedom since the intermediate nodes only forward the first copy of the same packet and discard the duplicate copies. To find a path to the destination, the source broadcasts a route request (RREQ) packet across the network. This RREQ contains the source identifier, the destination identifier, the source sequence number, the destination sequence number, the broadcast identifier, and the time to live field. Nodes that receive the RREQ find out whether they are the destination or whether they have a fresh route to the destination, then they respond to the RREQ by unicasting a route reply (RREP) back to the source node.

Otherwise, the node rebroadcasts the RREQ. When a node forwards an RREQ packet to its neighbors, it also records in its tables the node from which the first copy of the request came. This information is used to construct the reverse path for the RREP packet. AODV uses only 
symmetric links because the route reply packet follows the reverse path of route request packet. When a node receives an RREP packet, information about the previous node from which the packet was received is also stored in order to forward the data packets to this next node as the next hop toward the destination. Once the source node receives an RREP, it can begin using the route to send data packets. The source node rebroadcasts the RREQ if it does not receive an RREP before the timer expires. It attempts discovery up to some maximum number of attempts. If it does not discover a route after this maximum number of attempts, the session is aborted. If the source moves then it can reinitiate route discovery to the destination. If one of the intermediate nodes moves then the moved node neighbor realizes the link failure and sends a link failure notification to its upstream neighbors and so on till it reaches the source upon which the source can reinitiate route discovery if needed. The main advantage of AODV is that routes are obtained on demand and destination sequence numbers are used to find the latest route to the destination. One of the disadvantages of AODV is that intermediate nodes can lead to inconsistent routes if the source sequence number is very old and the intermediate nodes have a higher but not the latest destination sequence number, thereby causing stale entries. Also multiple route reply (RREP) packets in response to a single route request (RREQ) packet can lead to heavy control overhead. Periodic hello messages also lead to unnecessary bandwidth consumption.

\section{3. $D S R$}

The key feature of DSR $[10,13]$ is the use of source routing. That is, the sender knows the complete hop-by-hop route to the destination. These routes are stored in a route cache. The data packets carry the source route in the packet header. When a node in the ad hoc network attempts to send a data packet to a destination for which it does not already know the route, it uses a route discovery process to dynamically determine such a route. Route discovery works by flooding the network with route request (RREQ) packets. Each node receiving an RREQ rebroadcasts it, unless it is the destination or it has a route to the destination in its route cache. Such a node replies to the RREQ with a route reply (RREP) packet that is routed back to the original source. RREQ and RREP packets are also source routed. The RREQ builds up the path traversed so far. The RREP routes itself back to the source by traversing this path backwards, the route carried back by the RREP packet is cached at the source for future use. If any link on a source route is broken, the source node is notified using a route error (RERR) packet. The source removes any route using this link from its cache. A new route discovery process must be initiated by the source if this route is still needed. DSR makes very aggressive use of source routing and route caching. No special mechanism to detect routing loops is needed. Also, any forwarding node caches the source route in a packet it forwards for possible future use. Several additional optimizations have been proposed and have been evaluated to be very effective by the authors of the protocol [14], as described in the following. (i) Salvaging: an intermediate node can use an alternate route from its own cache, when a data packet meets a failed link on its source route. (ii) Gratuitous route repair: a source node receiving an RERR packet piggybacks the RERR in the following RREQ. This helps cleaning up the caches of other nodes in the network that may have the failed link in one of the cached source routes. (iii) Promiscuous listening: when a node overhears a packet not addressed to itself, it checks if the packet could be routed via itself to gain a shorter route. If so, the node sends a gratuitous RREP to the source of the route with this new, better route. Aside from this, promiscuous listening helps a node to learn different routes without directly participating in the routing process.

\section{CLASSIFICATION OF MOBILITY MODELS}

In general, the mobility models can be classified according to the different kinds of dependencies and restrictions that are considered.

(i) Random based: there are neither dependencies nor any other restrictions modeled which are similar to RWP model.

(ii) Temporal dependencies: the actual movement of a node is influenced by the movement of the past.

(iii) Spatial dependencies: the movement of a node is influenced by the nodes around it, for example, group mobility such as RPGM model.

(iv) Geographic restrictions: the area in which the node is allowed to move is restricted.

(v) Hybrid characteristics: a combination of temporal dependencies, spatial dependencies, and geographic restrictions is realized [15].

\section{MODELS}

\section{Random waypoint model}

The random waypoint mobility model is simple and is widely used to evaluate the performance of MANETs. The random waypoint mobility model contains pause time between changes in direction and/or speed. Once a mobile node (MN) begins to move, it stays in one location for a specified pause time. After the specified pause time is elapsed, the MN randomly selects the next destination in the simulation area and chooses a speed uniformly distributed between the minimum speed and maximum speed and travels with a speed $v$ whose value is uniformly chosen in the interval $\left(0, V_{\max }\right) . V_{\max }$ is some parameter that can be set to reflect the degree of mobility. Then, the MN continues its journey toward the newly selected destination at the chosen speed. As soon as the MN arrives at the destination, it stays again for the indicated pause time before repeating the process [16].

\section{Reference point group mobility model (RPGM)}

This model is described as another way to simulate group behavior in [17], where each node belongs to a group 
where every node follows a logical center (group leader) that determines the group's motion behavior. The nodes in a group are usually randomly distributed around the reference point. The different nodes use their own mobility model and are then added to the reference point which drives them in the direction of the group. At each instant, every node has a speed and direction that is derived by randomly deviating from that of the group leader. This general description of group mobility can be used to create a variety of models for different kinds of mobility applications. Group mobility as such can be used in military battlefield communications. One example of such mobility is that a number of soldiers may move together in a group. Another example is during disaster relief where various rescue crews (e.g., firemen, policemen, and medical assistants) form different groups and work cooperatively. The respective functions of group leaders and group members are described as follows.

\subsection{The group leader}

The movement of group leader at time, $t$, can be represented by motion vector $V_{\text {group }}^{t}$. Not only it defines the motion of group leader itself, but also it provides the general motion trend of the whole group. Each member of this group deviates from this general motion vector $V_{\text {group }}^{t}$ by some degree. The motion vector $V_{\text {group }}^{t}$ can be randomly chosen or carefully designed based on certain predefined paths [18].

\subsection{The group members}

The movement of group members is significantly affected by the movement of its group leader. For each node, mobility is assigned with a reference point that follows the group movement. Upon this predefined reference point, each mobile node could be randomly placed in the neighborhood.

Formally, the motion vector of group member, $i$, at time $t, V_{i}^{t}$, can be described as

$$
V_{i}^{t}=V_{\text {group }}^{t}+\mathrm{RM}_{i}^{t}
$$

where the motion vector $\mathrm{RM}_{i}^{t}$ is a random vector deviated by group member $i$ from its own reference point. The vector $\mathrm{RM}_{i}^{t}$ is an independent identically distributed random process whose length is uniformly distributed in the interval $\left[0, r_{\max }\right]$ (where $r_{\max }$ is maximum allowed distance deviation) and whose direction is uniformly distributed in the interval $[0,2 \pi]$.

$V_{\text {group }}^{t}$ is the motion vector for the group leader; it is also the motion vector for the whole group. $\mathrm{RM}_{i}^{t}$ is the random deviation vector for group member $i$, and the final motion vector of group member $i$ is represented by vector $V_{i}^{t}$.

With appropriate selection of predefined paths for group leader and other parameters, the RPGM model is able to emulate a variety of mobility behaviors. RPGM model is able to represent various mobility scenarios including the following. (i) In-place mobility model: the entire field is divided into several adjacent regions. Each region is exclusively occupied by a single group. One such example is battlefield communication.

(ii) Overlap mobility model: different groups with different tasks travel on the same field in an overlapping manner. Disaster relief is a good example.

(iii) Convention mobility model: this scenario is to emulate the mobility behavior in the conference. The area is also divided into several regions while some groups are allowed to travel between regions.

In RPGM model, the vector $\mathrm{RM}_{i}$ indirectly determine how much the motion of group members deviate from their leader. The movement can be characterized as follows:

$$
\begin{aligned}
\left|V_{\text {member }}(t)\right| & =\left|V_{\text {leader }}(t)\right|+\operatorname{random}() * \mathrm{SDR} * \text { max_speed } \\
\theta_{\text {member }}(t) & =\theta_{\text {leader }}(t)+\operatorname{random}() * \mathrm{SDR} * \max \_ \text {angle }
\end{aligned}
$$

(see [7]), where $0<\mathrm{SDR}, \mathrm{ADR}<1 . \mathrm{SDR}$ is the speed deviation ratio and $\mathrm{ADR}$ is the angle deviation ratio. SDR and $\mathrm{ADR}$ are used to control the deviation of the velocity (magnitude and direction) of group members from that of the leader. By simply adjusting these two parameters, different mobility scenarios can be generated.

Because of the inherent characteristic of spatial dependency between nodes, the RPGM model is expected to behave differently from the RWP model. We find that RPGM incurs less link breakage and achieves a better performance for various routing protocols than RWP model.

We observe that the mobility models have various properties and exhibit different mobility characteristics. As a consequence, we expect that mobility models behave differently and influence the protocol performance in different ways. Therefore, to thoroughly evaluate ad hoc protocol performance, it is imperative to use a rich set of mobility models. Each model has its own unique and specific mobility characteristics. Hence, a method to choose a suitable set of mobility models is needed.

\subsection{Simulation and performance metrics}

For our scenario-based experiments, we used the ns-2 simulator which is available as an open source distribution [19]. For generating the scenarios, we used the mobility scenario generation tool, BonnMotion. The radio model corresponds to the 802.11 WaveLAN, operating at a maximum airlink rate of $2 \mathrm{Mbps}$. The media access control protocol used is the IEEE 802.11 distributed coordination function (DCF). The traffic pattern file was generated using "cbrgen.tcl" script, which is provided along with the standard ns- 2 distribution. We used constant bit rate (CBR) traffic for our simulations.

\subsection{Metrics}

We have selected the packet delivery ratio, average end-toend delay, and protocol control overhead as a metric during 
TABLE 1: Parameters for RPGM and random waypoint model-mobility variations.

\begin{tabular}{lcc}
\hline Parameters & Values & Values \\
\hline Mobility model & RPGM & Random waypoint \\
Simulation area & $1000 \mathrm{~m} \times 1000 \mathrm{~m}$ & Uniform speed \\
Minimum node speed & $1 \mathrm{~m} / \mathrm{s}$ & 2000 \\
\# of nodes & 20 & $\mathrm{CBR}$ \\
Traffic type & CBR (constant bit rate) & 512 bytes \\
Packet size & 512 bytes & 10 pkts/s \\
Connection rate & 10 pkts/s & $25 \mathrm{~s}$ \\
Pause time & $25 \mathrm{~s}$ & 5 \\
Number of connections & 5 & $10-15-20-25 \mathrm{~m} / \mathrm{s}$ \\
Maximum node speed & $10-15-20-25 \mathrm{~m} / \mathrm{s}$ & 200 \\
Simulation time & $200 \mathrm{~s}$ & - \\
Distribution of nodes & in each group 10 groups & AODV, DSR \\
Protocols & AODV, DSR & - \\
Probability of group change & 0.05 & - \\
Maximum distance to group center & $100 \mathrm{~m}$ & - \\
Standard deviation & 2.0 & -
\end{tabular}

TABLE 2: Parameters for RPGM and random waypoint-node density variations.

\begin{tabular}{lcc}
\hline Parameters & Values & Values \\
\hline Mobility model & RPGM & Random waypoint \\
Simulation area & $1000 \mathrm{~m} \times 1000 \mathrm{~m}$ & $1000 \mathrm{~m} \times 1000 \mathrm{~m}$ \\
Minimum node speed & $1 \mathrm{~m} / \mathrm{s}$ & Uniform speed \\
Number of nodes & $20,50,80,100$ & $20,50,80,100$ \\
Traffic type & CBR (constant bit rate $)$ & CBR \\
Packet size & 512 bytes & 512 bytes \\
Connection rate & $20 \mathrm{pkts} / \mathrm{s}$ & $20 \mathrm{pkts} / \mathrm{s}$ \\
Pause time & $20-30 \mathrm{~s}$ & $25 \mathrm{~s}$ \\
Number of connections & 5 & 5 \\
Maximum node speed & $10 \mathrm{~m} / \mathrm{s}$ & $10 \mathrm{~m} / \mathrm{s}$ \\
Simulation time & $200 \mathrm{~s}$ & $200 \mathrm{~s}$ \\
Protocols & AODV, DSR & AODV, DSR \\
Distribution of nodes & 2 in each group 10 groups & - \\
Probability of group change & 0.05 & - \\
Maximum distance to group center & $100 \mathrm{~m}$ & - \\
Standard deviation & 2.0 & -
\end{tabular}

the simulation in order to evaluate the performance of the different protocols [20].

(a) Packet delivery fraction $(P D F)$ : this is the ratio of total number of packets successfully received by the destination nodes to the number of packets sent by the source nodes throughout the simulation:

$$
\mathrm{PDF}=\frac{\text { number of received packets }}{\text { number of sent packets }} .
$$

This estimate gives us an idea about how successful the protocol is in delivering packets to the application layer. A high value of PDF indicates that most of the packets are being delivered to the higher layers and is a good indicator of the protocol performance. (b) Normalized routing load (NRL): this is calculated as the ratio between the number of routing packets transmitted to the number of packets actually received (thus accounting for any dropped packets):

$$
\mathrm{NRL}=\frac{\text { number of routing packets sent }}{\text { number of data packets received }} .
$$

This metric gives an estimate of how efficient a routing protocol is since the number of routing packets sent per data packet gives an idea of how well the protocol maintains the routing information updated. The higher the NRL, the higher the overhead of routing packets and consequently the lower the efficiency of the protocol is. 


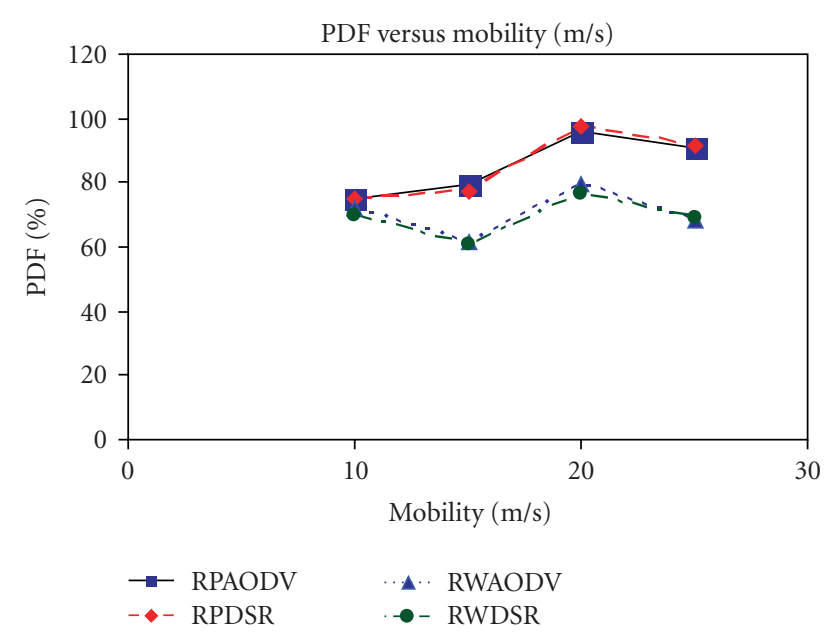

(a)

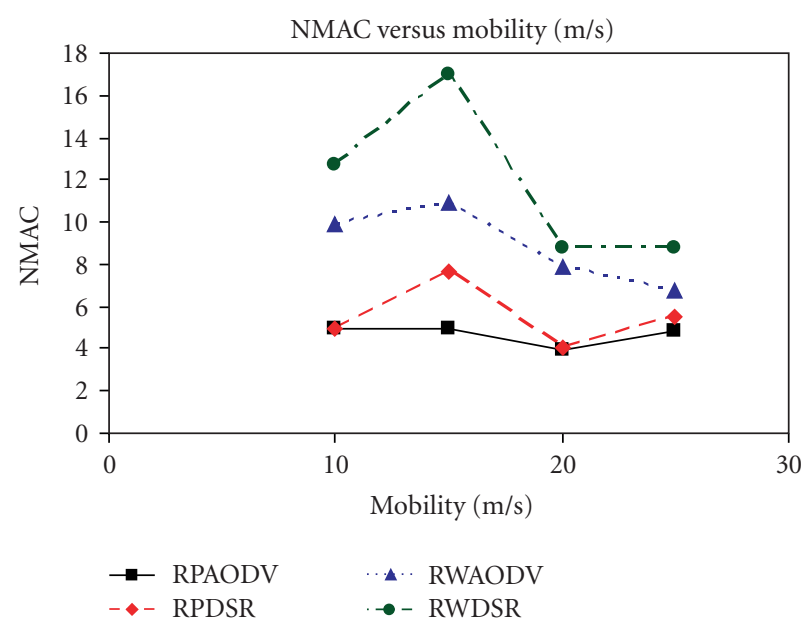

(c)

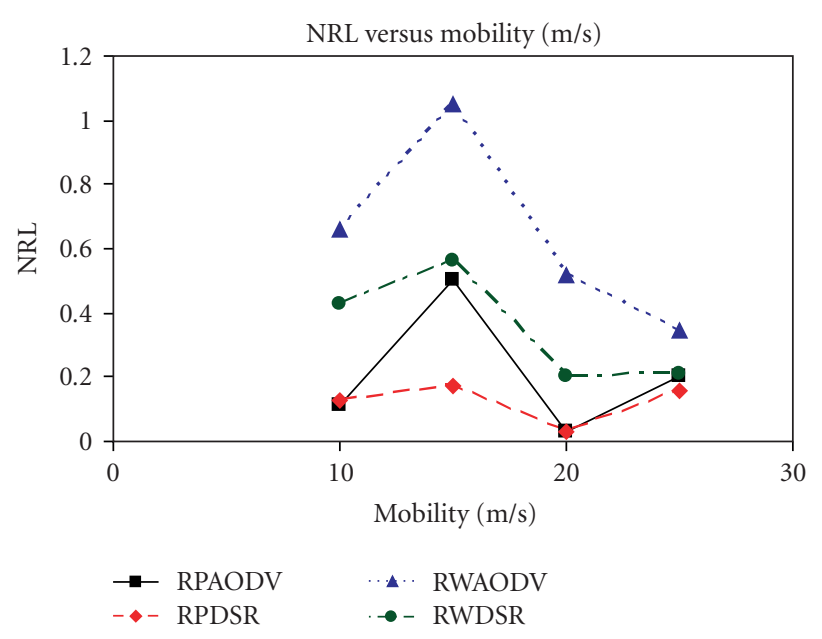

(b)

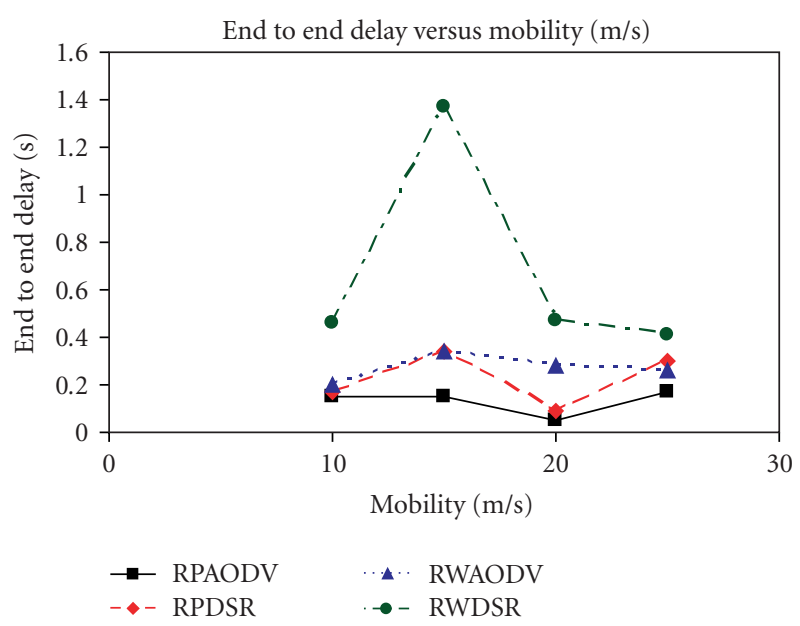

(d)

FIGURE 1

TABLE 3: Parameters for RPGM and random waypoint-network loading.

\begin{tabular}{lcc}
\hline Parameters & Values & Values \\
\hline Mobility model & RPGM & Random waypoint \\
Simulation area & $1000 \mathrm{~m} \times 1000 \mathrm{~m}$ & $1000 \mathrm{~m} \times 1000 \mathrm{~m}$ \\
Minimum node speed & $1 \mathrm{~m} / \mathrm{s}$ & Uniform speed \\
Number of nodes & 20 & 20 \\
Traffic type & CBR (constant bit rate) & 512 bytes \\
Packet size & 512 bytes & $20-30-40-50-60$ pkts/s \\
Connection rate & $20-30-40-50-60$ pkts/s & $25 \mathrm{~s}$ \\
Pause time & $20-30 \mathrm{~s}$ & 5 \\
Number of connections & 5 & $10 \mathrm{~m} / \mathrm{s}$ \\
Maximum node speed & $10 \mathrm{~m} / \mathrm{s}$ & $200 \mathrm{~s}$ \\
Simulation time & $200 \mathrm{~s}$ & - \\
Distribution of nodes & 2 in each group 10 groups & - \\
Probability of group change & 0.05 & - \\
Maximum distance to group center & $100 \mathrm{~m}$ & - \\
Standard deviation & 2.0 & -
\end{tabular}




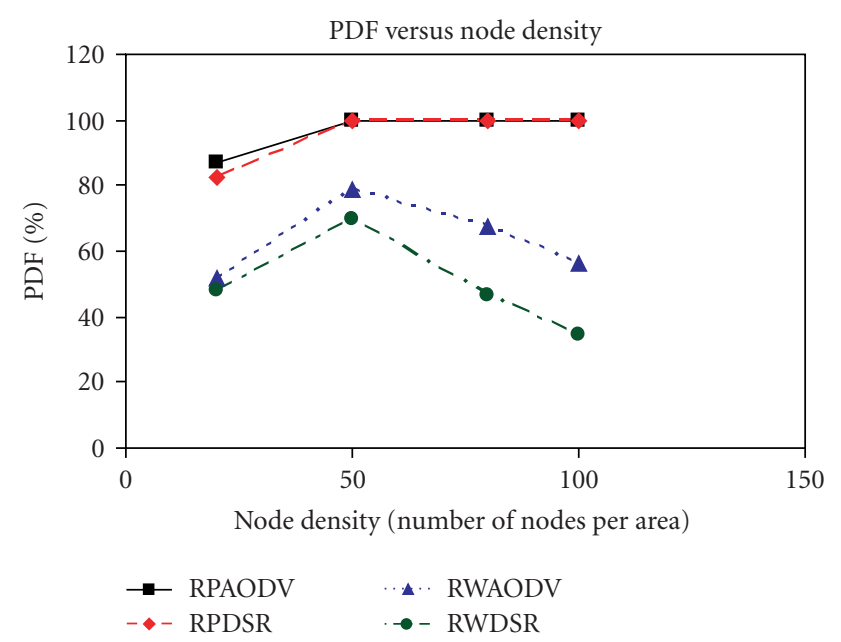

(a)

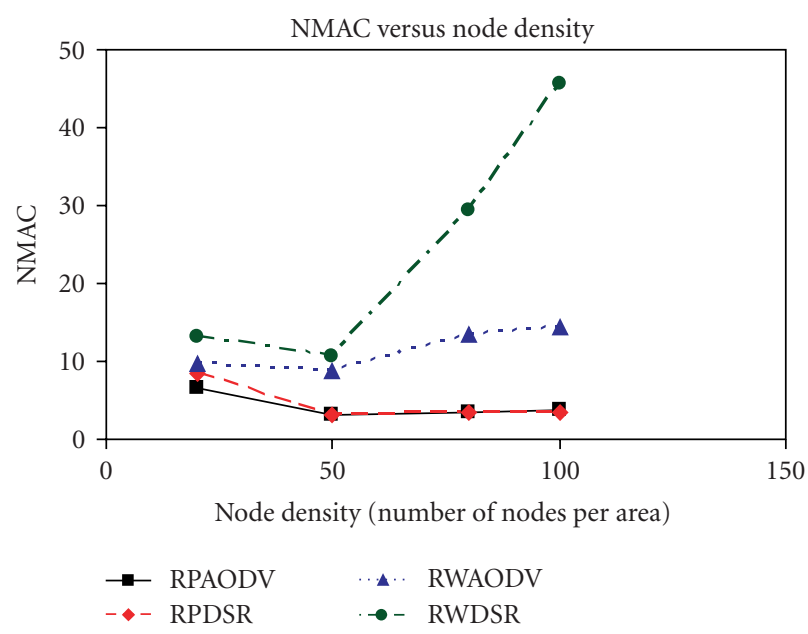

(c)

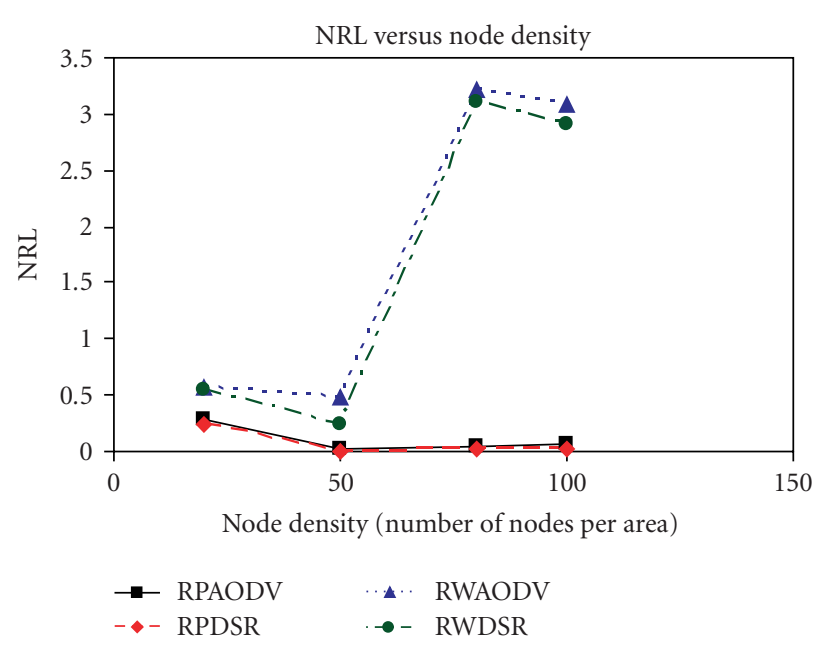

(b)

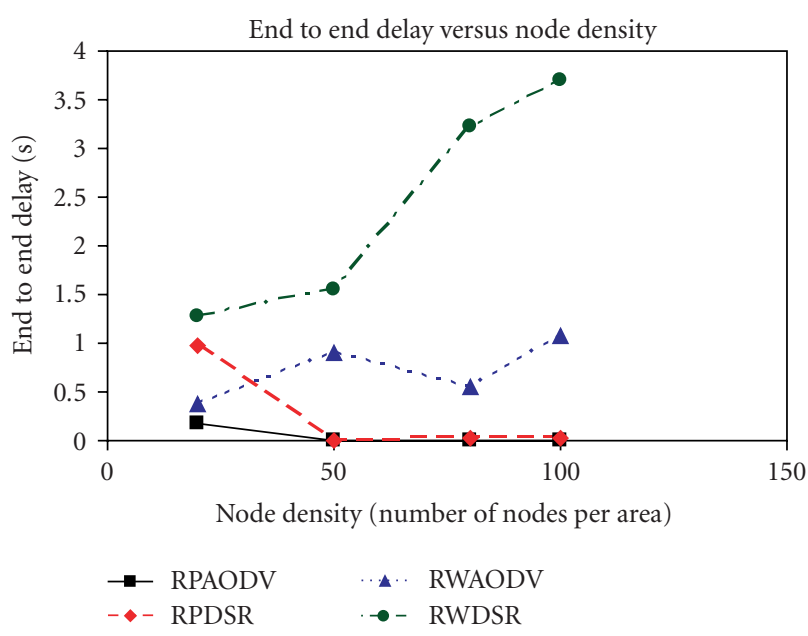

(d)

Figure 2

(c) Average end-to-end delay (AED): this is defined as the average delay in transmission of a packet between two nodes and is calculated as follows:

$\mathrm{AED}=\sum_{i=0}^{n} \frac{\left(\text { time packet received }_{i}-\text { time packet sent } \mathrm{t}_{i}\right)}{\text { total number of packets received }}$

A higher value of end-to-end delay means that the network is congested and hence the routing protocol does not perform well. The upper bound on the values of end-to-end delay is determined by the application.

\section{DESCRIPTION OF THE SCENARIOS}

We consider different scenarios for our experiments, in which the nodes are distributed over the simulation area.
The scenarios depict varying node densities, link changes, and mobility variations. To thoroughly study the effect of mobility on MANET protocol performance, we need to evaluate the protocols over a rich set of mobility models that span the design space of the mobility metrics. The scenarios are explained in the following sections.

The reference point group mobility (RPGM) model [21] is used for modeling the scenario. In RPGM model, a cluster of nodes communicates in groups. The velocity and direction of nodes within the group are determined by a "group leader" or reference point. Since the group leader mainly decides the mobility of group members, group mobility pattern is expected to have high spatial dependence for small values of SDR and ADR. We define the parameters in this mobility model as shown in Table 1.

In random waypoint model, a mobile node chooses a random destination at every instance and moves toward it with a speed uniformly distributed $\left[0, V_{\max }\right]$, where $V_{\max }$ is the maximum allowable speed for a node. After reaching the 


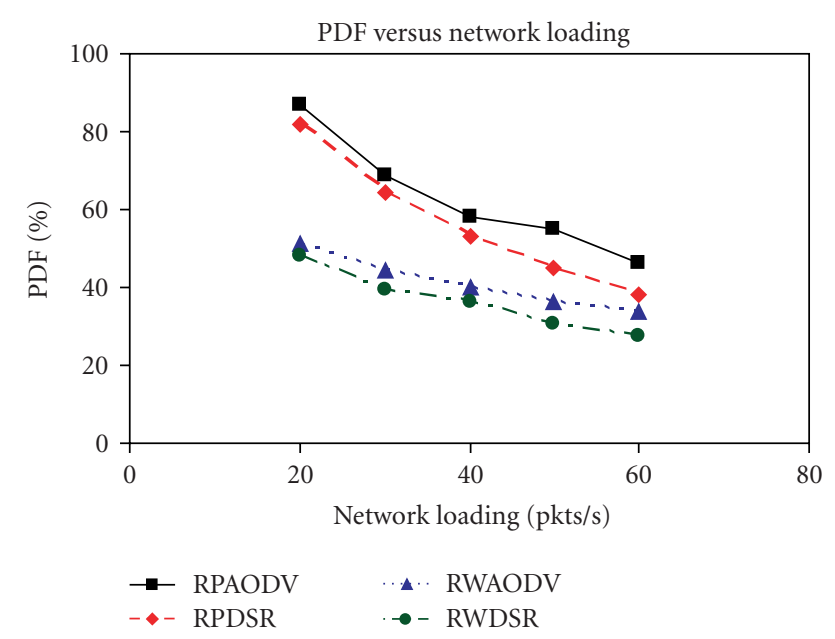

(a)

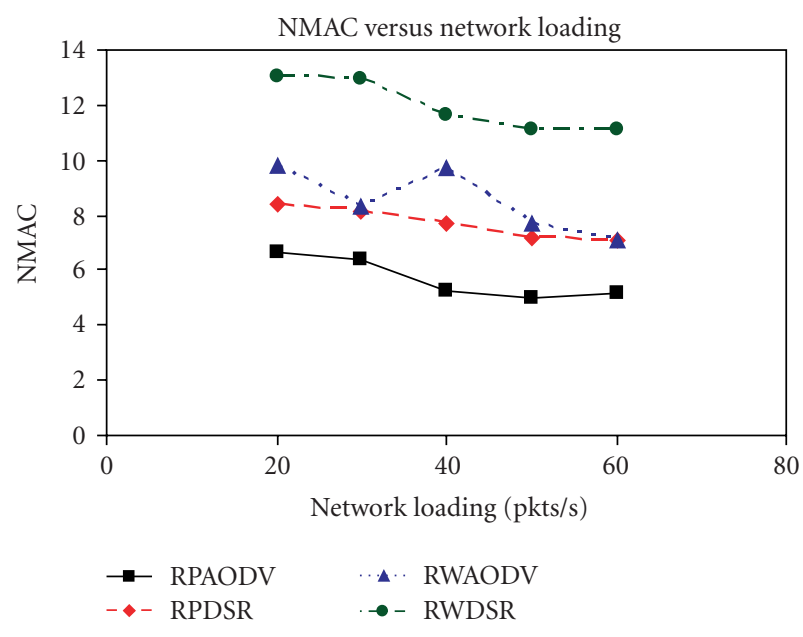

(c)

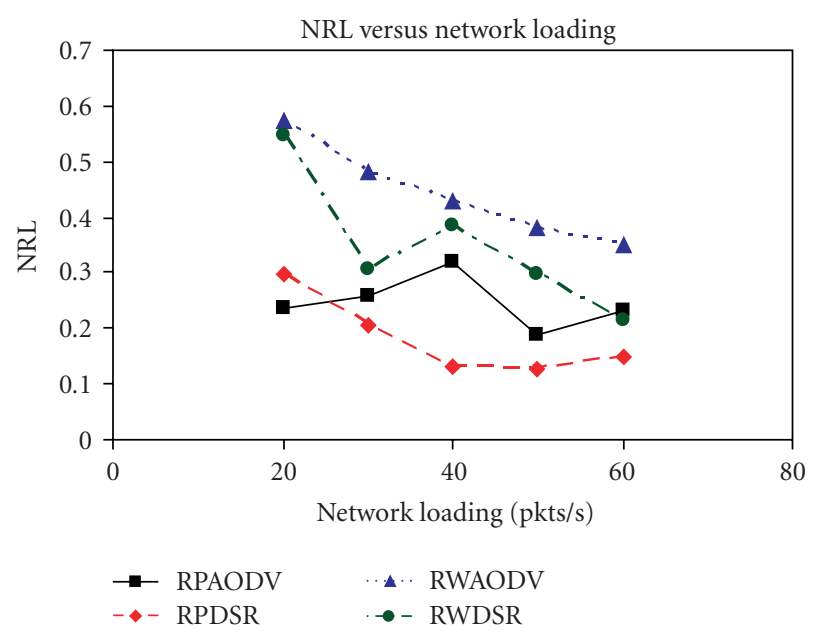

(b)

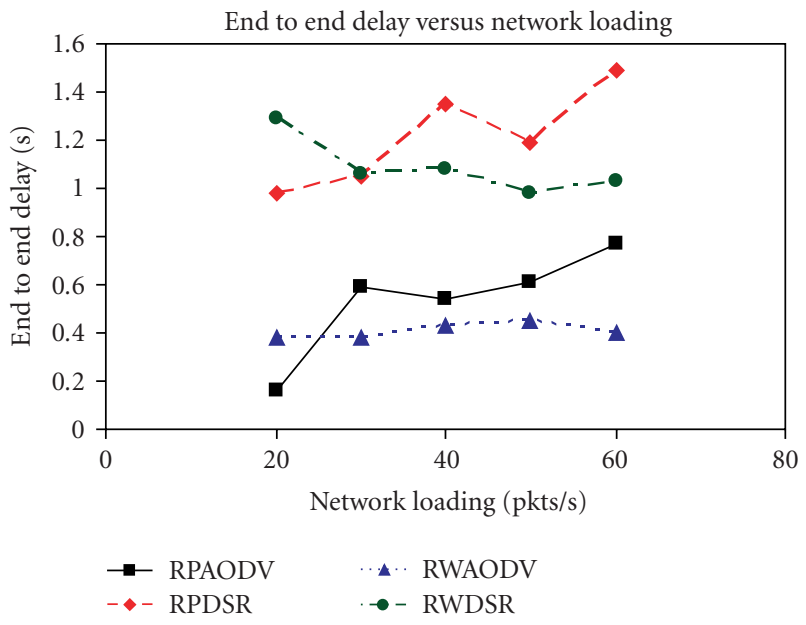

(d)

Figure 3

destination, the node stops for a duration defined by the "pause time" parameter. After this duration, it again chooses a random destination and repeats the whole process again until the simulation ends.

\subsection{Mobility variations results}

The analysis of Figure 1(a) shows that AODV and DSR have similar performance outputs. From Figure 1(b), we infer that routing overheads generated by AODV is greater than DSR. This can be attributed to more routing updates needed in AODV. DSR uses source routing and also caches some routing entries. The normalized medium access control (MAC) load for AODV is found to be less when compared to DSR. This is because route errors (RERRs) (Figure 1(c)) are handled differently in each protocol. RERRs are unicast in DSR and therefore contribute to additional MAC overhead like route replies (RREPs). In AODV, RERRs are broadcast like route requests (RREQs) and hence are less expensive. Consequently, when the MAC overhead is factored, DSR is found to generate higher overall network load than AODV in all scenarios despite having less routing overhead. The end-to-end delay of AODV is less when compared to DSR (Figure 1(d)).

\subsection{Node density results}

In the second experiment on RPGM model, we try to find the performance aspect when the node density is varied within a fixed map area. The node density is incrementally adjusted from 20-100 nodes within the same map area of $1000 \times 1000 \mathrm{~m}$. The simulation parameters are shown in Table 2. From Figure 2(a), we infer that DSR and AODV have similar results. The routing overhead remains relatively low for DSR whereas for AODV it increases tremendously. The network delay of AODV is less when compared to DSR. 


\subsection{Network loading results}

In the third set of experiments of RPGM, we try to find the routing performance aspect when the offered load increases. To do this, we increase the average connection load offered by each connection starting at 20-60 pkts/s. The same parameters are used, such as the type of traffic is constant bit rate (CBR) and the nodes are pumping data as shown in Table 3. Figure 3(a) shows that the PDF of routing protocols have very close performance results when the network loading is high. Overall, the protocols experience a drop in packet delivery ratio as network loading increases. Figure 3(b) shows that DSR has less overhead when compared to AODV. Figure 3(c) shows that DSR has a high-normalized MAC load when compared to AODV. We see from Figure 3(d) that AODV has lowest network latency in the RPGM model when network loading increases compared to DSR.

\section{CONCLUSION}

(1) In this study, analysis has been made on the impact of mobility pattern on routing performance of mobile ad hoc network in a systematic manner.

(2) It has been observed that the mobility pattern influences the performance of MANET routing protocols. There is a very clear trend between mobility metric, connectivity, and performance.

(3) It has been observed that with similar average spatial dependency when the relative speed increases, the link duration decreases and hence the routing overhead increases and throughput decreases.

(4) With similar average relative speed, it is found that spatial dependence increases and the link duration increases and therefore, the throughput increases and the routing overhead decreases.

(5) It has been observed that DSR and AODV achieve the highest throughput and least overhead with RPGM when compared to RWP mobility models. This is because with similar relative speed, between random waypoint and RPGM, high degree of spatial dependence for RPGM means higher link duration and correspondingly higher path duration, which in turn will result in higher throughput and lower routing overhead.

(6) RWP model is insufficient to capture the following mobility characteristics such as temporal dependence and spatial dependence. This reduces the throughput when compared to RPGM.

(7) From the results, it is analyzed that AODV has better throughput and less delay in RPGM model when compared to RWP model. For circumstances such as military operation, AODV may emerge as a better choice.

(8) Thus, it is necessary to conclude that relative rankings of the protocols may vary with the mobility model used. This clearly shows that mobility pattern influences the connectivity graph which in turn influences the protocol performance.

\section{ACKNOWLEDGMENT}

The authors would like to thank the anonymous reviewers for their careful reading and insightful comments that have helped in improving the presentation of this paper.

\section{REFERENCES}

[1] I. Awan and K. Al-Begain, "Performance evaluation of wireless networks," International Journal of Wireless Information Networks, vol. 13, no. 2, pp. 95-97, 2006.

[2] S. Yi, Y. Pei, S. Kalyanaraman, and B. Azimi-Sadjadi, "How is the capacity of ad hoc networks improved with directional antennas?" Wireless Networks, vol. 13, no. 5, pp. 635-648, 2007.

[3] S. L. Kota, E. Hossain, R. Fantacci, and A. Karmouch, "Crosslayer protocol engineering for wireless mobile networks-part 1," IEEE Communications Magazine, vol. 43, no. 12, pp. 110$111,2005$.

[4] S. L. Kota, E. Hossain, R. Fantacci, and A. Karmouch, "Crosslayer protocol engineering for wireless mobile networks-part 2," IEEE Communications Magazine, vol. 44, no. 1, pp. 83-84, 2006.

[5] D. R. Grinath and S. Selvan, "Performance analysis of dispersion mobility model in mobile ad hoc networks," International Journal of Computer Science and Network Security, vol. 8, no. 3, pp. 77-87, 2008.

[6] R. Folio, J. B. Cain, and S. Kota, "Challenges in the verification of mobile ad hoc networking systems," International Journal of Wireless Information Networks, vol. 14, no. 2, pp. 107-120, 2007.

[7] G. Lin, G. Noubir, and R. Rajaraman, "Mobility models for ad hoc network simulation," in Proceedings of the 23rd Annual Joint Conference of the IEEE Computer and Communications Societies (INFOCOM '04), vol. 1, pp. 454-463, Hongkong, March 2004.

[8] E. Hyytiä and J. Virtamo, "Random waypoint mobility model in cellular networks," Wireless Networks, vol. 13, no. 2, pp. 177188, 2007.

[9] P. S. Mogre, M. Hollick, N. d'Heureuse, H. W. Heckel, T. Krop, and R. Steinmetz, "A graph-based simple mobility model," in Proceedings of the 4th Workshop on Mobile AdHoc Networks (WMAN '07), pp. 421-432, Bern, Switzerland, February-March 2007.

[10] D. B. Johnson and D. Maltz, "Dynamic source routing in ad hoc wireless networks," in Mobile Computing, T. Imielinski and H. Korth, Eds., pp. 153-181, Kluwer Academic Publishers, Dordrecht, The Netherlands, 1996.

[11] C. E. Perkins and E. M. Royer, "Ad hoc on-demand distance vector routing," in Proceedings of the 2nd IEEE Workshop on Mobile Computing Systems and Applications (WMCSA '99), pp. 90-100, New Orleans, La, USA, February 1999.

[12] J. Broch, A. D. Maltz, D. B. Johnson, Y.-C. Hu, and J. G. Jetcheva, "A performance comparison of multi-hop wireless ad hoc network routing protocols," in Proceedings of the 4th Annual ACM/IEEE International Conference on Mobile Computing and Networking (MobiCom '98), pp. 85-97, Dallas, Tex, USA, October 1998. 
[13] J. Broch, D. Johnson, and D. Maltz, "The dynamic source routing protocol for mobile ad hoc networks," IETF Internet Draft, December 1998, http://www.ietf.org/internet-drafts/ draft-ietf-manet-dsr-11.txt.

[14] D. A. Maltz, J. Broch, J. Jetcheva, and D. B. Johnson, "The effects of on-demand behavior in routing protocols for multihop wireless ad hoc networks," IEEE Journal on Selected Areas in Communications, vol. 17, no. 8, pp. 1439-1453, 1999.

[15] N. Aschenbruck, E. Gerhards-Padilla, and P. Martini, "A survey on mobility models for performance analysis in tactical mobile networks," Journal of Telecommunications and Information Technology, vol. 2, pp. 54-61, 2008.

[16] I. Khider, W. Furong, and Y. Wei, "Study on indoor and outdoor environment for mobile ad hoc network supported with base sstations," in Proceedings of the International Conference on Wireless Communications, Networking and Mobile Computing (WiCom '07), pp. 1470-1474, Shanghai, China, September 2007.

[17] X. Hong, M. Gerla, G. Pei, and C. C. Chiang, "A group mobility model for ad hoc wireless networks," in Proceedings of the 2nd ACM International Workshop on Modeling, Analysis and Simulation of Wireless and Mobile Systems (MSWiM '99), pp. 53-60, Seattle, Wash, USA, August 1999.

[18] F. Bai, N. Sadagopan, and A. Helmy, "Important: a framework to systematically analyze the impact of mobility on performance of routing protocols for ad hoc networks," in Proceedings of the 22nd Annual Joint Conference on the IEEE Computer and Communications Societies (INFOCOM '03), vol. 2, pp. 825-835, San Francisco, Calif, USA, March 2003.

[19] K. Fall and K. Varadhan, "The NS Manual," The VINT Project, UC Berkeley, January 2002.

[20] S. Gowrishankar, T. G. Basavaraju, and S. K. Sarkar, "Effect of random mobility models pattern in mobile ad hoc networks," International Journal of Computer Science and Network Security, vol. 7, no. 6, pp. 160-164, 2007.

[21] T. Camp, J. Boleng, and V. Davies, "A survey of mobility models for ad hoc network research," Wireless Communications and Mobile Computing, vol. 2, no. 5, pp. 483-502, 2002. 

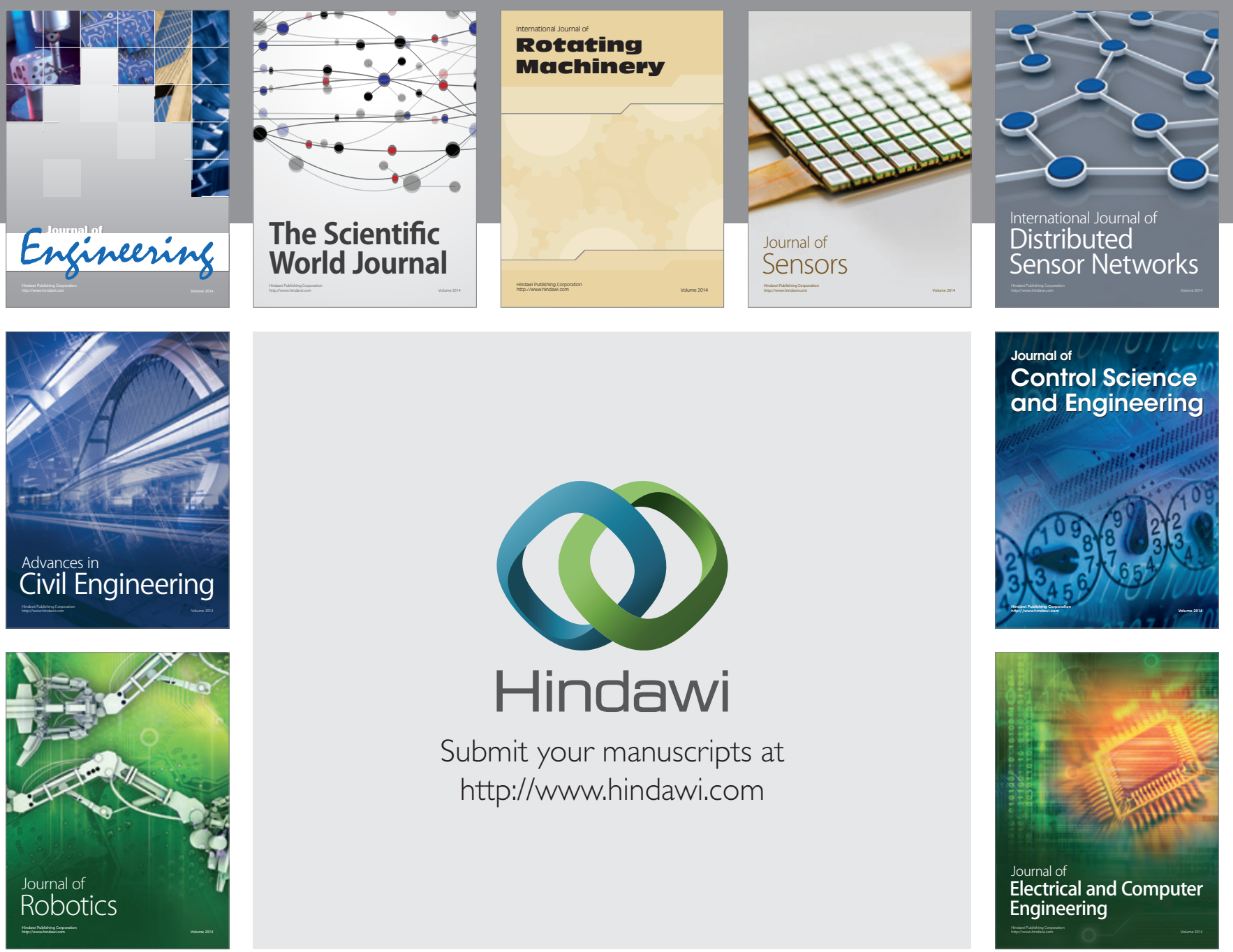

Submit your manuscripts at

http://www.hindawi.com
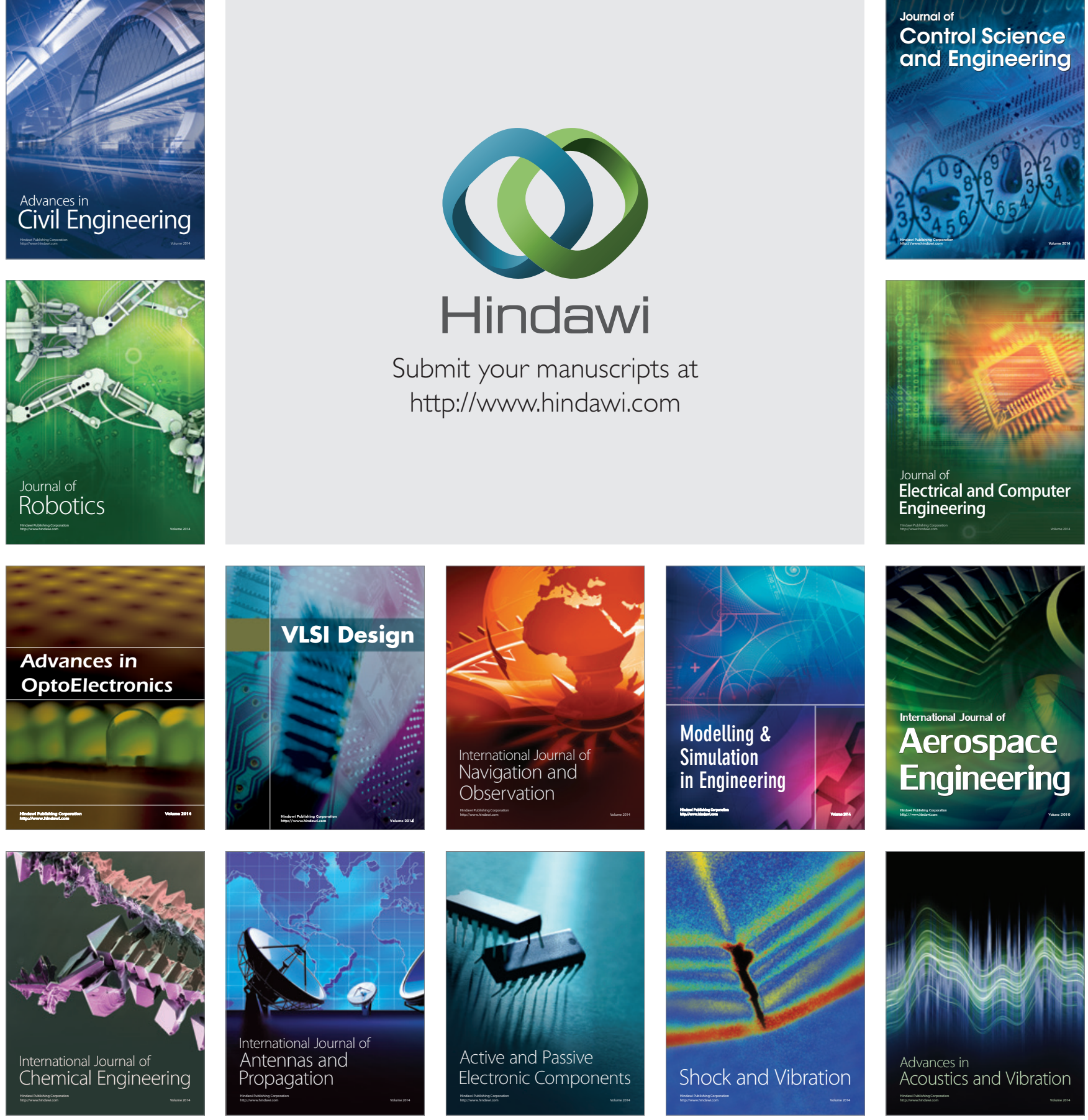